\section{Synchrotron-based Spectroscopy Reveals First Evidence for Organic Sulfur-coordinated Arsenic in Peat}

\section{Peggy Langner, Christian Mikutta*,} and Ruben Kretzschmar

${ }^{*}$ Correspondence: Dr. C. Mikutta, Institute of Biogeochemistry and Pollutant Dynamics, Soil Chemistry Group, ETH Zurich, Universitätsstrasse 16, CH-8092 Zurich, Tel.: +414463360 24, Fax: +41446331118,

E-mail: christian.mikutta@env.ethz.ch

Keywords: Arsenic · Iron · Natural organic matter · Peatland Sulfur $\cdot$ X-ray absorption spectroscopy

Arsenic is recognized as a toxic contaminant and a threat to some of the world's water resources. Accumulating in organicrich soils and sediments, As is typically associated with $\mathrm{Fe}$ oxyhydroxide, silicate, and sulfide minerals. Natural organic matter (NOM) has also been suggested as a potential sorbent for As, but the binding mechanisms are still elusive. Since our understanding of the biogeochemical As cycle is therefore essentially incomplete, the major goal of this study was to (1) identify the major mechanism of As binding to NOM and (2) assess its quantitative importance relative to well-established As sequestration pathways involving mineral phases. To this end, we studied the solid-phase speciation of As and $\mathrm{Fe}$ in a groundwaterfed peatland (Gola di Lago, canton Ticino, Switzerland) containing high natural As concentrations. Anoxic peat samples were analyzed by bulk As and Fe K-edge X-ray absorption near edge structure (XANES) and extended X-ray absorption fine structure (EXAFS) spectroscopy. We focused our speciation analyses on shallow $(<0.4 \mathrm{~m})$ and deep $(1.5-2.6 \mathrm{~m})$ peat layers.

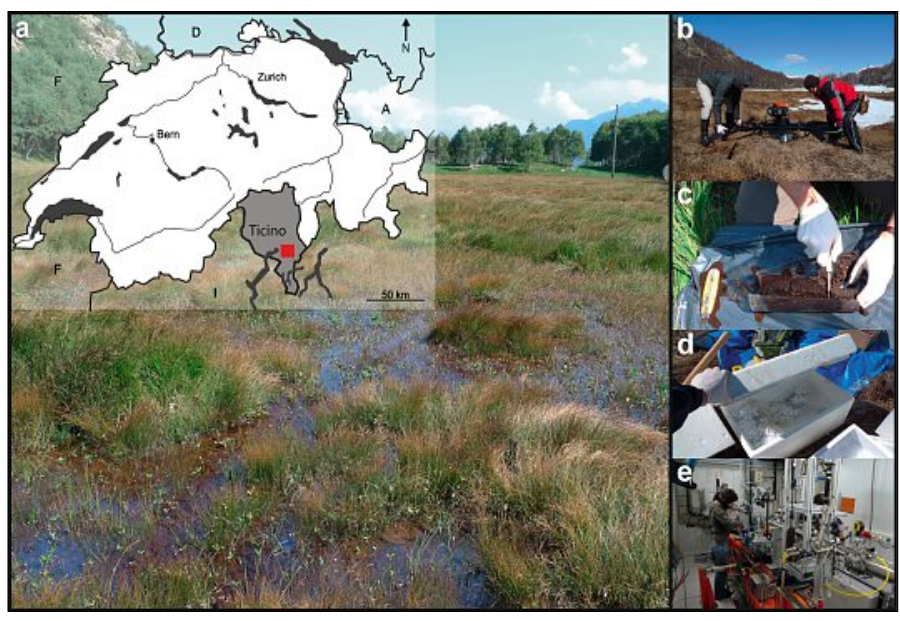

Fig. 1. The field site Gola di Lago (red square) is situated in canton Ticino, Switzerland (a). In order to preserve the element speciation in the samples, retrieved peat cores were rapidly cut into slices, shockfrozen, and stored on dry ice for transportation to the laboratory (b-d). After anoxic sample preparation, selected samples were measured at beamline BM29 of the European Synchrotron Radiation Facility (ESRF, Grenoble, France) (e).

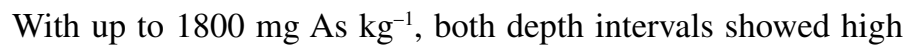
As concentrations but differed significantly in the contents of $\mathrm{Fe}$ and sulfur (S). Speciation analyses of As revealed that close to the peat surface As was mainly present as As sulfide, and As(III)/(v) sorbed to Fe(III)-oxyhydroxides. In the deep peat layers, however, As was almost entirely coordinated in its trivalent oxidation state to 2-3 $\mathrm{S}$ atoms of NOM. The Fe speciation was dominated by chlorite, Fe(III)-oxyhydroxides, Fe(III)-NOM complexes, and Fe sulfides. Although the latter three phases were shown or hypothesized to immobilize As(III), nearly all As was associated with NOM.

Our results thus imply that the binding of As to sulfhydryl groups of NOM impairs As sequestration pathways involving reactive $\mathrm{Fe}$ species, and suggest that this mechanism is the major mode of As-NOM interactions in moderately anoxic and S-rich environments.

\section{Acknowledgements}

We acknowledge the European Synchrotron Radiation Facility in Grenoble, France (ESRF) for provision of synchrotron radiation facilities, and would like to thank $M$. Chorro for assistance in using beamline BM29.

\section{Reference}

Received: August 22, 2012

P. Langner, C. Mikutta, R. Kretzschmar, Nature Geoscience 2012, 5, 66.

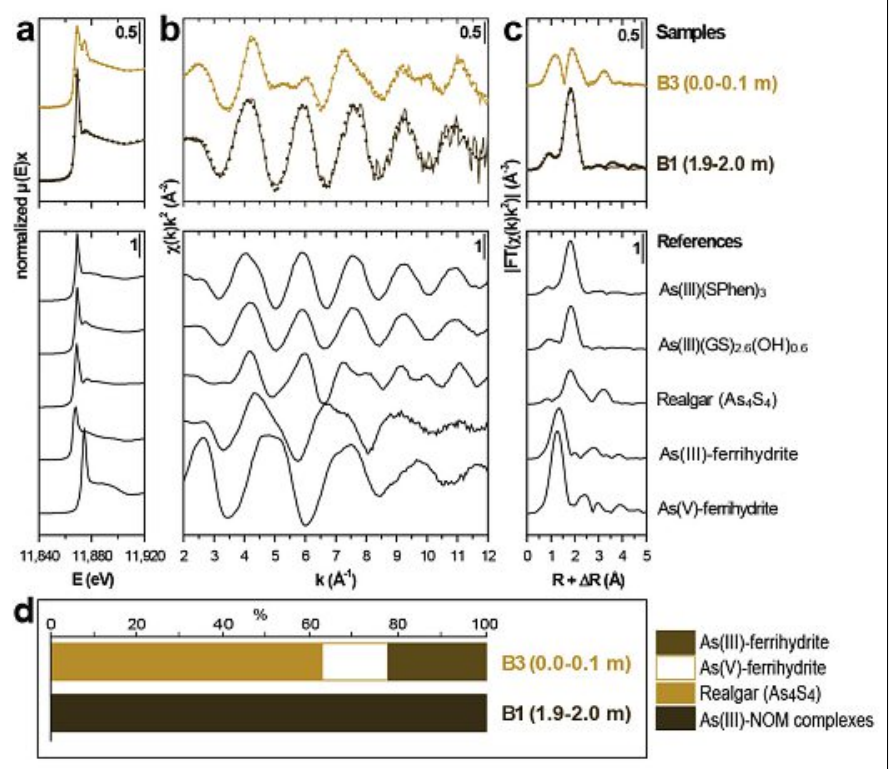

Fig. 2. Arsenic K-edge XANES (a) and $k^{2}$-weighted EXAFS spectra (b) as well as Fourier-transform magnitudes (c) of two representative peat samples and selected reference compounds. The displayed reference spectra were used to model the EXAFS spectra of the peat samples. Solid lines are experimental data and filled circles represent best model fits. Results of the As speciation analysis are summarized in (d). Tris(phenyl)thioarsine (As(III)(SPhen) ${ }_{3}$ ) and a mixture of di- and tri(glutamylcysteinylglycinyl)-thioarsenite $\left(\mathrm{As}(\mathrm{III})(\mathrm{GS})_{2.6}(\mathrm{OH})_{0.6}\right)$ were used as references for organic S-coordinated As(III). 